VoL. 51 (1995) [25-30]

\title{
A NOTE ON STRONG DITKIN ALGEBRAS
}

\author{
J.F. FEINSTEIN
}

\begin{abstract}
In this note we answer a question of W. G. Bade by showing that if a normal, unital Banach function algebra $A$ is strongly regular at one of its characters $\phi$, and the kernel of $\phi$ has a bounded approximate identity, then $A$ has bounded relative units at $\phi$. In particular, every strong Ditkin algebra has bounded relative units at all points of its character space. There need not, however, be a global bound available for the relative units.
\end{abstract}

\section{TERMINOLOGY AND NOTATION}

In our terminology a compact space is a compact, Hausdorff topological space. For any compact space $X$ we denote by $C(X)$ the algebra of all continuous, complex-valued functions on $X$. For a set $E \subseteq X$ we denote the characteristic function of $E$ by $\chi_{E}$.

Let $A$ be a commutative, unital algebra. We denote by $\Phi_{A}$ the character space of $A$, and by $\operatorname{Inv}(A)$ the set of invertible elements of $A$.

DEFINITION 1: Let $X$ be a compact space. A function algebra on $X$ is a subalgebra of $C(X)$ which contains the constant functions and separates the points of $X$. A Banach function algebra on $X$ is a function algebra on $X$ with a complete algebra norm. A uniform algebra on $X$ is a Banach function algebra on $X$ whose norm is the uniform norm on $X,\|\cdot\|_{\infty}$. A function algebra $A$ on $X$ is self-adjoint if, for all $f \in A$, the conjugate function

$$
x \mapsto \overline{f(x)}
$$

is also in $A$.

We shall restrict our attention to unital Banach algebras. Every commutative, semisimple Banach algebra can be regarded, via the Gelfand transform, as a Banach function algebra on its character space.

Notation. Let $A$ be a Banach function algebra on $\Phi_{A}$. Let $\phi \in \Phi_{A}$. We define the ideals $J_{\phi}, M_{\phi}$ as follows:

$$
\begin{gathered}
M_{\phi}=\{f \in A: f(\phi)=0\} \\
J_{\phi}=\left\{f \in A: f^{-1}(\{0\}) \text { is a neighbourhood of } \phi\right\} .
\end{gathered}
$$

Received 29th August, 1994

Copyright Clearance Centre, Inc. Serial-fee code: 0004-9729/95 \$A2.00+0.00. 
Definition 2: Let $A$ be a Banach function algebra on $\Phi_{A}$. Then $A$ is normal if, for every pair of disjoint closed sets $E, F$ contained in $\Phi_{A}$, there is an $f \in A$ with $f(E) \subseteq\{0\}$ and $f(F) \subseteq\{1\}$.

Let $\phi \in \Phi_{A}$. Then $A$ is strongly regular at $\phi$ if $J_{\phi}$ is dense in $M_{\phi}: A$ satisfies the Ditkin condition at $\phi$ if for all $f \in M_{\phi}$ and all $\varepsilon>0$ there exists $g \in J_{\phi}$ with $\|g f-f\|<\varepsilon$. We say that $A$ has bounded relative units at $\phi$ if there is a $C \geqslant 1$ such that, for all compact sets $E \subseteq \Phi_{A} \backslash\{\phi\}$, there is $f \in J_{\phi}$ with $\|f\| \leqslant C$ such that $f(E) \subseteq\{1\}$.

The algebra $A$ is strongly regular if it is strongly regular at every point; $A$ is a Ditkin algebra if it satisfies Ditkin's condition at every point; $A$ is a strong Ditkin algebra if it is strongly regular, and every maximal ideal has a bounded approximate identity.

It is clear that every Ditkin algebra is strongly regular.

There are two definitions in print for a Banach function algebra $A$ to have bounded relative units. One is that, for every $\phi \in \Phi_{A}, A$ has bounded relative units at $\phi$. The other definition is stronger, insisting that the constant $C$ involved does not depend on $\phi$. It is not clear that these two definitions differ: indeed for uniform algebras they are equivalent. But we shall give an example to show that these definitions are different for general Banach function algebras.

The following result is well known. The proof is identical with that of the corresponding result for uniform algebras [2, Lemma 1 (iii)].

Proposition 3. Let $A$ be a Banach function algebra on $\Phi_{A}$. Let $\phi \in \Phi_{A}$. Suppose that $A$ is strongly regular at $\phi$ and $M_{\phi}$ has a bounded approximate identity. Then $M_{\phi}$ has a bounded approximate identity consisting of elements of $J_{\phi}$.

Note in particular that every strong Ditkin algebra is indeed a Ditkin algebra. In [1], Bade asked the following question: If $A$ is a strong Ditkin algebra, must $A$ have bounded relative units at every point of its character space? We resolve this question positively. In fact we answer the corresponding question about individual points of the character space.

Theorem 4. Let $A$ be a normal Banach function algebra on $\Phi_{A}$. Let $\phi \in \Phi_{A}$. Suppose that $A$ is strongly regular at $\phi$ and that $M_{\phi}$ has a bounded approximate identity. Then $A$ has bounded relative units at $\phi$.

Proof: By Proposition 3, $M_{\phi}$ has a bounded approximate identity $\left(e_{\nu}\right)$ consisting of elements of $J_{\phi}$. Set $C=\sup _{\nu}\left\|e_{\nu}\right\|$. Let $\varepsilon>0$. We show that $A$ has bounded relative units at $\phi$ with bound $C+\varepsilon$. To see this, let $E$ be a compact subset of $\Phi_{A} \backslash\{\phi\}$. Choose $g \in J_{\phi}$ such that $g(E) \subseteq\{1\}$. Then the functions $1-\left(1-e_{\nu}\right)(1-g)$ are also in $J_{\phi}$ 
and are identically 1 on $E$. But

$$
\left\|1-\left(1-e_{\nu}\right)(1-g)\right\|=\left\|e_{\nu}+g-e_{\nu} g\right\| \leqslant C+\left\|g-e_{\nu} g\right\| .
$$

Choosing $\nu$ such that $\left\|g-e_{\nu} g\right\|<\varepsilon$, set $f=e_{\nu}+g-e_{\nu} g$. Then $f \in J_{\phi},\|f\| \leqslant C+\varepsilon$ and $f(E) \subseteq\{1\}$.

Of course it now follows that every strong Ditkin algebra must have bounded relative units at every point of its character space. We now give an example to show that there need not exist any global bound: the bound may genuinely depend on the point chosen. In particular this example will have the property that every maximal ideal has a bounded approximate identity, but no global constant can work simultaneously for all points.

Definition 5: Throughout the rest of this paper, $\mathbb{N}_{\infty}$ will denote the one point compactification of $\mathbb{N}$. We define

$$
A_{1}=\left\{f \in C\left(\mathbb{N}_{\infty}\right): \sum_{n=1}^{\infty}|f(n+1)-f(n)|<\infty\right\} .
$$

For each $k \in \mathbb{N}$ we define a seminorm $p_{k}$ and a norm $\|\cdot\|_{k}$ on $A_{1}$ by

$$
\begin{gathered}
p_{k}(f)=k \sum_{n=1}^{\infty}|f(n+1)-f(n)|, \\
\|f\|_{k}=\|f\|_{\infty}+p_{k}(f) .
\end{gathered}
$$

Clearly $A_{1}$ is a Banach function algebra under any of the equivalent norms $\|\cdot\|_{k}$. We now use these norms to build up our counterexample. Set

$$
A_{2}=\left\{g \in A_{1}{ }^{\mathbf{N}}: \lim _{k \rightarrow \infty}\left\|g_{k}\right\|_{k}=0\right\}
$$

the $c_{0}$ sum of the algebras $\left(A_{1},\|\cdot\|_{k}\right)$. Then $A_{2}$ is a (non-unital) commutative Banach algebra, which has unitisation

$$
A_{2}^{\#}=\left\{g \in A_{1}{ }^{\mathbf{N}}: \text { there exists } \lambda \in \mathbb{C} \text { with } \lim _{k \rightarrow \infty}\left\|g_{k}-\lambda\right\|_{k}=0\right\},
$$

on which we can place the complete norm

$$
\|g\|=\sup _{k \in \mathbb{N}}\left\|g_{k}\right\|_{k}
$$

The algebra $A_{2} \#$ can be identified with a Banach function algebra on a countable, compact subset of $\mathbb{R}$ : choose $a_{k, n} \in \mathbb{R}(k, n \in \mathbb{N})$ such that

$$
1 /(k+1)<a_{k, 1}<a_{k, 2}<\cdots<1 / k
$$


and such that $a_{k, n} \rightarrow 1 / k$ as $n \rightarrow \infty$ (for example we may take $a_{k, n}=1 / k-$ $\left.1 /\left(n(k+1)^{2}\right)\right)$. Set

$$
X=\{0\} \cup\left\{1, \frac{1}{2}, \frac{1}{3}, \cdots\right\} \cup \bigcup_{k=1}^{\infty}\left\{a_{k, 1}, a_{k, 2}, a_{k, 3}, \cdots\right\}
$$

( $X$ is homeomorphic to the one point compactification of a countable disjoint union of copies of $\left.\mathbb{N}_{\infty}\right)$. We have (surjective) homomorphisms $\pi_{k}: C(X) \rightarrow C\left(\mathbb{N}_{\infty}\right)$ defined by $\pi_{k}(h)(n)=h\left(a_{k, n}\right), \pi_{k}(h)(\infty)=h(1 / k)$. It is clear that $A_{2}{ }^{\#}$ is isometrically isomorphic to the Banach function algebra

$$
B=\left\{h \in C(X): \pi_{k}(h) \in A_{1} \text { for all } k \in \mathbb{N} \text { and } \lim _{k \rightarrow \infty} p_{k}\left(\pi_{k}(h)\right)=0\right\}
$$

with the norm

$$
\|h\|=\sup _{k \in \mathbf{N}}\left\|\pi_{k}(h)\right\|_{k}=\sup _{k \in \mathbb{N}}\left(\sup _{n \in \mathbb{N}}\left|h\left(a_{k, n}\right)\right|+k \sum_{n=1}^{\infty}\left|h\left(a_{k, n+1}\right)-h\left(a_{k, n}\right)\right|\right) .
$$

We shall see that this algebra $B$ has the desired properties. We begin by showing that the only characters on $B$ are evaluations at the points of $X$. To see this we use the following result.

Proposition 6. $[4$, p.55] Let $A$ be a function algebra on a compact space $X$. Suppose that $A$ is self-adjoint, and that $\operatorname{Inv}(A)=A \cap \operatorname{Inv}(C(X))$. Then the only characters on $A$ are evaluations at points of $X$.

LEMMA 7. The only characters on $B$ are the evaluations at points of $X$.

Proof: Clearly $B$ is self-adjoint. Now suppose that $h \in B \cap \operatorname{Inv}(C(X))$. Then set

$$
\delta=\inf _{x \in X}|h(x)|
$$

We have $\delta>0$, and

$$
\begin{aligned}
p_{k}\left(\pi_{k}\left(h^{-1}\right)\right) & =k \sum_{n=1}^{\infty}\left|\frac{1}{h\left(a_{k, n+1}\right)}-\frac{1}{h\left(a_{k, n}\right)}\right| \\
& \leqslant \delta^{-2} k \sum_{n=1}^{\infty}\left|h\left(a_{k, n+1}\right)-h\left(a_{k, n}\right)\right| \\
& =\delta^{-2} p_{k}\left(\pi_{k}(h)\right)
\end{aligned}
$$

which tends to 0 as $k \rightarrow \infty$. This shows that $h^{-1} \in B$. 
Thus $\operatorname{Inv}(B)=B \cap \operatorname{Inv}(C(X))$, and so the only characters on $B$ are evaluations at points of $X$.

Next we show that $B$ is strongly regular, and, in fact, for every $x \in X, J_{x}$ contains a bounded approximate identity for $M_{x}$.

When $x=a_{n, k}$ we have $J_{x}=M_{x}$, so $1-\chi_{\{x\}}$ is in $J_{x}$ and is an identity for $M_{x}$.

For $x=k^{-1}$, to see that $J_{x}$ contains a bounded approximate identity for $M_{x}$, set

$$
E_{n}=\left\{k^{-1}, a_{k, n}, a_{k, n+1}, \cdots\right\} .
$$

Then we may take $e_{n}=1-\chi E_{n}$ as our bounded approximate identity.

Finally, for $x=0, J_{0}$ contains a bounded approximate identity for $M_{0}$, namely $e_{n}=1-\chi_{X \cap\left[0, n^{-1}\right]}$.

Thus $B$ is a strong Ditkin algebra, and so has bounded relative units at every point. But any collection of bounded relative units at the point $a_{k, 2}$ must include the function $1-\chi_{\left\{a_{k, 2}\right\}}$, whose norm is $2 k+1$.

Putting all this together gives us the desired result.

THEOREM 8 . There exists a strong Ditkin algebra for which there is no possible global bound for the relative units.

\section{OPEN QUESTIONS}

The relationships between the various regularity conditions for Banach function algebras are now fairly well understood. Most of the remaining open questions in this area concern uniform algebras. Here the two definitions of bounded relative units are equivalent, and a uniform algebra has bounded relative units if and only if it is a strong Ditkin algebra. Examples in [3] show that a uniform algebra which is strongly regular need not be a strong Ditkin algebra, and there are uniform algebras other than $C(X)$ which are strong Ditkin algebras. The following questions appear to be open.

(1) Is every strongly regular uniform algebra a Ditkin algebra?

(2) Is every uniform algebra which is a Ditkin algebra also a strong Ditkin algebra?

The examples mentioned above show that (1) and (2) can not both have positive answers. There are also versions of (1) and (2) asked at one point at a time. In [5], Wang gave an example of a compact set $X \subseteq \mathbb{C}$ with $0 \in X$ such that $R(X)$ is strongly regular at 0 but $M_{0}$ does not have a bounded approximate identity. (Here $R(X)$ is the uniform algebra of all $f \in C(X)$ which may be uniformly approximated by rational functions with no poles in $X$ ). One specific question is the following.

(3) Does Wang's example satisfy Ditkin's condition at the point 0 ? 
Many questions about $R(X)$ remain open, including the following.

(4) Does there exist a compact plane set $X$ such that $R(X)$ is strongly regular, but $R(X) \neq C(X)$ ?

Two similar questions which are still of interest both in the general case and for uniform algebras are the following.

(5) Let $A$ be a normal Banach function algebra on $\Phi_{A}$, and let $\phi \in \Phi_{A}$. Suppose that $M_{\phi}$ has a bounded approximate identity. Does it follow that $A$ must be strongly regular at $\phi$ ?

(6) Let $A$ be a normal Banach function algebra on $\Phi_{A}$, and suppose that, for all $\phi \in \Phi_{A}, M_{\phi}$ has a bounded approximate identity. Does it follow that $A$ must be strongly regular?

In [3] it is shown that (5) and (6) must have the same answer for uniform algebras.

\section{REFERENCES}

[1] W.G. Bade, 'Open problems', in Conference on Automatic Continuity and Banach Algebras, Proceedings of the Centre for Mathematical Analysis 21 (Australian National University, 1989).

[2] D.R. Chalice, 'S-algebras on sets in $\mathbb{C}^{n}$, ', Proc. Amer. Math. Soc. 39 (1973), 300-304.

[3] J.F. Feinstein, 'A non-trivial, strongly regular uniform algebra', J. London Math. Soc. 45 (1992), 288-300.

[4] L.H. Loomis, An introduction to abstract harmonic analysis (van Nostrand, New York, 1953).

[5] J.L-M. Wang, 'Strong regularity at nonpeak points', Proc. Amer. Math. Soc. 51 (1975), 141-142.

Department of Mathematics

The University of Nottingham

Nottingham NG7 2RD

England

e-mail: jff@maths.nottingham.ac.uk 